ÉGYPTE monde arabe

\section{Égypte/Monde arabe}

2 | 2005

Les architectures constitutionnelles des régimes

politiques arabes

\title{
Monarchies constitutionnelles et républiques non constitutionnelles
}

Mécanismes juridiques de succession dans le monde arabe moderne

\section{Nathan Brown}

Traducteur : Cécile Lagoutte et Nathalie Bernard-Maugiron

\section{CpenEdition}

\section{Journals}

Édition électronique

URL : https://journals.openedition.org/ema/1061

DOI : 10.4000/ema.1061

ISSN : 2090-7273

Éditeur

CEDEJ - Centre d'études et de documentation économiques juridiques et sociales

Édition imprimée

Date de publication : 31 décembre 2005

Pagination : 89-104

ISSN : 1110-5097

Référence électronique

Nathan Brown, « Monarchies constitutionnelles et républiques non constitutionnelles », Égypte/Monde arabe [En ligne], 2 | 2005, mis en ligne le 08 juillet 2008, consulté le 07 juillet 2022. URL : http:// journals.openedition.org/ema/1061; DOI : https://doi.org/10.4000/ema.1061

Ce document a été généré automatiquement le 7 juillet 2022

Tous droits réservés 


\title{
Monarchies constitutionnelles et républiques non constitutionnelles
}

\author{
Mécanismes juridiques de succession dans le monde arabe moderne
}

\author{
Nathan Brown \\ Traduction : Cécile Lagoutte et Nathalie Bernard-Maugiron
}

1 Depuis plus de trois siècles, les systèmes politiques du Moyen-Orient sont considérés par les auteurs occidentaux comme des exemples de gouvernement arbitraire. La gouvernance dans les sociétés moyen-orientales, contrairement à l'État de droit, est considérée comme s'exerçant dans le cadre d'un pouvoir absolu et selon le caprice personnel. Pour de nombreux fondateurs de la tradition libérale occidentale, la pratique gouvernementale orientale a été un repoussoir bien utile. Les régimes ottoman et persan étaient présentés comme des modèles négatifs présentant toutes les caractéristiques d'un despotisme sans limites. Cela ne pouvait mieux s'observer que dans le domaine de la succession. Montesquieu affirmait que le résultat n'était pas seulement malheureux pour les sujets, mais fâcheux pour l'État lui-même :

Dans les États où il n'y a point de lois fondamentales, la succession à l'empire ne saurait être fixe. La couronne y est élective par le prince, dans sa famille, ou hors de sa famille. En vain serait-il établi que l'aîné succéderait; le prince en pourrait toujours choisir un autre. Le successeur est déclaré par le prince lui-même, ou par ses ministres, ou par une guerre civile. Ainsi cet État a une raison de dissolution de plus qu'une monarchie.

Chaque prince de la famille royale ayant une égale capacité pour être élu, il arrive que celui qui monte sur le trône fait d'abord étrangler ses frères, comme en Turquie; ou les fait aveugler, comme en Perse; ou les rend fous, comme chez le Mogol, chaque vacance de trône est suivie d'une affreuse guerre civile. (Montesquieu, 1951, p. 295-296)

2 Les auteurs du xIX et du début du Xxe siècle ont continué de dépeindre la pratique du pouvoir arabe - et moyen-oriental de façon plus générale - comme un pur despotisme. Ceux qui colonisèrent la région évoquèrent fréquemment la difficulté de rendre la justice dans une région qui n'avait connu que la règle de l'arbitraire. Dans les années récentes, ces clichés dominants ne se sont pas estompés mais sont devenus plus subtils. 
Le monde arabe est souvent décrit comme une région restée à l'abri des vagues de démocratisation qui ont submergé d'autres régions.

3 Les récits d'yeux crevés et de fratricide rapportés par Montesquieu sont rarement repris lorsqu'on évoque le manque de démocratie dans la région. Les termes ont évolué, et les anciens dirigeants "despotiques» sont devenus "autoritaires». Mais l'image sous-jacente d'arbitraire et d'irresponsabilité perdure. Ceci ne signifie pas que les auteurs contemporains ne font que reproduire d'anciens préjugés. Une telle accusation serait incorrecte pour deux raisons.

4 Premièrement, le comportement politique des gouvernants arabes vient étayer dans une large mesure de telles images. Deuxièmement, l'évolution de la terminologie de "despotisme" à «autoritarisme" marque une transformation, voire un approfondissement, de l'ancien cynisme concernant la politique dans la région. Les auteurs classiques et les premiers penseurs libéraux établirent une distinction entre les gouvernements autocratiques, qui suivaient des modèles juridiques clairs, et les gouvernements qui le ne faisaient pas (suivant la citation précédente, la distinction se fait entre monarques et despotes). Cette distinction s'est perdue. Tous les autocrates paraissent désormais semblables. C'est en partie dû aux définitions de plus en plus ambitieuses et étendues de la démocratie, qui n'ont fait qu'accroitre la liste des échecs politiques arabes. De plus en plus, le discours savant, comme celui du plus grand public, ne définit plus seulement la démocratie comme le gouvernement par le peuple, mais comme le gouvernement conformément aux principes libéraux. Les fondateurs de la tradition politique libérale - bien plus méfiants à l'égard de la démocratie que les auteurs contemporains - n'auraient pas fait aussi facilement que ces derniers l'amalgame entre démocratie et État de droit. Cet amalgame atteint son apogée sur la question de la désignation des dirigeants. Selon ces définitions de plus en plus procédurales, un trait distinctif de la démocratie est par exemple le caractère incertain du résultat. En d'autres termes, il n'y a pas de démocratie - quels que soient les mécanismes formels observés - si les dirigeants actuels sont assurés de leur propre maintien en fonction ou de l'identité de leurs successeurs. Adam Przeworski, par exemple, distingue entre gouvernement arbitraire et gouvernement démocratique, non pas en termes de souveraineté populaire mais en termes d'incertitude institutionnalisée des résultats politiques. Il écrit :

le moment crucial, dans tout passage de l'autoritarisme à la démocratie, n'est pas forcément le retour de l'armée dans les casernes, ou l'ouverture du Parlement élu, mais le passage du seuil au-delà duquel personne ne peut plus intervenir pour renverser les résultats du processus démocratique formel. (Przeworski, 1988, p. 62)

5 L'alternative de Przeworski à la démocratie - l'autoritarisme - est, elle aussi, définie explicitement en termes de procédure : l'autoritarisme existe s'il y a « un appareil de pouvoir susceptible de renverser les résultats du processus politique institutionnalisé » (id. p 60).

6 L'effet de ces conceptions novatrices de la démocratie est pour le monde arabe de rappeler l'éternelle dichotomie : d'un côté se tient le gouvernement libéral et l'État de droit, de l'autre se dresse le despotisme et le règne de l'arbitraire. L'ajout du concept de démocratie a approfondi la dichotomie: il y surimpose les pôles opposés de responsabilité populaire et de pouvoir absolu. Dans les systèmes politiques arabes, les responsables sont imposés aux peuples, plutôt que choisis par eux. 
7 Cette image de la politique arabe est de plus en plus répandue dans la région ellemême. En 1965, la pièce à succès de Tawfîq al-Hakîm « Le dilemme du sultan » (1998) montrait un souverain contraint de choisir entre la légalité ou la poursuite de son propre règne. La critique implicite des souverains contemporains était assez douce. Aujourd'hui, les intellectuels arabes seraient bien plus durs. Peu contesteraient l'idée que les gouvernements actuels sont anarchiques, despotiques et irresponsables.

Cette image de la pratique gouvernementale arabe a de solides fondements. Mais l'opposition totale qu'elle présente entre autoritarisme et État de droit peut induire en erreur, parce qu'elle passe sous silence le mélange de légalité et d'autoritarisme qui émerge - avec vigueur - dans les politiques arabes. État de droit et autoritarisme ne s'excluent pas mutuellement: ces dernières années ils se sont souvent renforcés. Autocratie et constitutionnalisme sont dans le monde arabe aussi souvent associés que dissociés. On peut l'observer dans de nombreux domaines, mais peut-être jamais aussi clairement qu'à propos des mécanismes de succession. L'un des principaux attraits des « lois fondamentales » ou des constitutions réside en fait dans leur capacité à orienter les processus de succession.

9 Les régimes monarchiques dans le monde arabe inscrivent souvent les procédures de succession dans les textes constitutionnels. C'est particulièrement vrai pour les pays qui ne sont pas simplement gouvernés par des monarques seuls, mais par des familles régnantes (comme les États de la Péninsule arabique, à l'exception du Yémen) ${ }^{1}$. Les souverains qui usent d'un tel dispositif ont en général deux objectifs. D'abord, la succession constitutionnelle limite généralement les luttes et les discordes au sein de la famille royale. Ensuite, les constitutions arabes concentrent en général l'autorité sur le processus de succession entre les mains du souverain régnant. Elles affermissent par-là à la fois sa place en ce monde, et son héritage au seuil de l'autre monde. L'observation des procédures de succession dans les monarchies du Golfe confirme cette analyse.

10 Étrangement, les systèmes politiques les plus profondément fondés sur les formes politiques occidentales - les républiques arabes - ont des procédures constitutionnelles de succession moins développées. En Syrie, en 2000, la Constitution fut taillée sur mesure pour la succession (plutôt que l'inverse) et uniquement après la mort du président. En Irak, aucune succession constitutionnelle ne s'est produite depuis presque un demi-siècle; en Égypte et dans l'Autorité palestinienne, les actuels gouvernants ont fait preuve d'une profonde méfiance envers les mécanismes constitutionnels. Et même lorsque ceux-ci sont utilisés par des républiques, les résultats tendent à être plus contestés et ambigus que lorsqu'ils sont utilisés dans les monarchies.

\section{Successions constitutionnelles dans les monarchies arabes}

11 Alors que le monde arabe a une réputation d'instabilité politique, la longévité des régimes actuels est remarquable. En un sens, les monarchies de la péninsule arabique ne sont pas différentes: toutes peuvent faire remonter leurs règnes à un siècle au moins. Pourtant, alors que les régimes monarchiques sont stables, les monarques, en tant qu'individus, ne le sont pas : même lorsque la monarchie elle-même n'a pas été contestée, le règne des monarques a souvent été plus bref que les charges à vie d'autres 
chefs d'État de la région. Des six monarques du Golfe actuellement régnants, par exemple, deux sont parvenus à leur poste en renversant leurs propres pères.

Le Qatar a connu deux coups d'État depuis son indépendance (et un contre coup d'État manqué), l'Arabie saoudite a vu l'un de ses rois déposé dans les années 1960 et un autre assassiné dans les années 1970. Le sultan d'Oman a été déposé en 1970. Dans trois autres États, pourtant, les monarques n'ont quitté leur poste que lorsqu'ils ont reçu l'appel du Très-Haut. Deux des émirs du Koweït sont morts depuis l'indépendance, et le pouvoir a été immédiatement transféré à leur successeur désigné. La succession à Bahreïn s'est déroulée selon le même schéma en 2003. Les Émirats arabes unis sont dirigés par le même souverain depuis l'indépendance, bien que les Émirats membres aient connu des transitions.

Deux caractéristiques des modèles de succession dans la péninsule méritent une attention particulière. Premièrement, dans tous les cas, les successions irrégulières ont été provoquées par des divisions au sein de la famille royale. Les transitions sans heurts se sont produites le plus souvent quand la question de la succession était clairement réglée (souvent spécifiée au moment de l'accession au pouvoir du nouveau monarque). Les monarchies du Golfe, en général, ne sont pas absolutistes: il existe quelques contrôles formels du pouvoir des souverains, mais les familles régnantes sont vastes, puissantes, exigeantes et parfois querelleuses. Des souverains sont morts des mains de leurs proches (au Koweït en 1896 et en Arabie saoudite en 1975), pas des mains de leurs autres sujets.

Deuxièmement, les transitions sans heurts ont été bien plus courantes dans les pays disposant de procédures constitutionnelles claires réglant la question. L'Arabie saoudite a connu deux successions irrégulières sans constitution, la famille régnante koweïtienne - aussi âprement divisée qu'une autre - a suivi chaque fois les procédures constitutionnelles. En résumé, les familles régnantes rendent la succession difficile et les constitutions aident à soulager ces difficultés.

$15 \mathrm{Au}$ xIXe siècle, les souverains arabes du Golfe parvinrent à différents accords avec la Grande-Bretagne, qui firent de leurs pays des protectorats de fait. Les Britanniques garantirent généralement l'autorité de la famille régnante, en échange du contrôle sur les affaires étrangères et la sécurité. Dans un cas - celui du Qatar - les Britanniques reconnurent non seulement l'émir de manière explicite, mais aussi la succession de son fils. Avec le retrait britannique du Golfe en 1971, de tels arrangements prirent fin. D'une certaine façon, on peut alors considérer les constitutions de la région comme des équivalents fonctionnels de la Royal Navy.

Certes, les documents écrits peuvent s'avérer moins efficaces que les navires de guerre, mais ils sont beaucoup plus efficaces que ce à quoi l'on pouvait s'attendre au départ. Les expériences du Koweït et de Bahreïn le prouvent.

\section{Koweït}

17 Le Koweït accéda à l'indépendance en 1961. L'émir de l'époque, cAbd Allah Salîm, convoqua une assemblée constituante. Les membres élus furent rejoints par des ministres (issus de la famille régnante). L'émir travailla avec quelques experts constitutionnels égyptiens pour présenter un projet à l'Assemblée. Un comité spécial de l'Assemblée révisa le projet, puis le soumit en plénière pour approbation². 
18 La Constitution koweïtienne est toujours en vigueur à ce jour (même si le Parlement qu'elle a créé a été suspendu deux fois). Le document est remarquable par le degré d'attention qu'il porte au rôle de la famille régnante, aussi bien qu'aux relations à l'intérieur de celle-ci. Tout d'abord, les membres de la famille royale ne peuvent être élus au Parlement, mais ils y sont représentés par des membres de la famille royale nommés ministres. Cette procédure n'est pas stipulée formellement dans la Constitution, mais elle est reconnue de façon implicite. Par tradition, aucun des membres de la famille Âl Sabah ne s'est porté candidat aux élections au Parlement, mais les ministres peuvent être choisis en dehors des cinquante membres de l'Assemblée, et y siéger en vertu de leur statut ministériel. Le nombre de ministres ne doit pas dépasser un tiers de l'Assemblée. Les membres de la famille Âl Sabah ont occupé des postes administratifs de haut rang et ont souvent monopolisé les ministères clés. Grâce à leurs fonctions de ministres, de nombreux membres de la famille régnante ont ainsi pu accéder à des postes de pouvoir. Puisque c'est le Premier ministre (en pratique, toujours le prince héritier) qui choisit les ministres et qui est responsable devant l'émir (bien qu'il le soit aussi, dans une moindre mesure, devant le Parlement), la domination de l'émir et de son successeur désigné sur le reste de la famille est ainsi accrue.

19 La Constitution régit aussi de façon explicite le processus de succession lui-même : l'émir présente le prince héritier qu'il a choisi au Parlement pour ratification. L'article 4 de la Constitution du Koweït stipule :

Le dauphin sera désigné un an au plus tard à partir de la date de l'accession au trône de l'émir. Sa désignation sera effectuée par un ordre émiral sur nomination de l'émir, et avec l'approbation de l'Assemblée nationale qui devra être signifiée par un vote à la majorité de ses membres en séance spéciale. Au cas où aucune désignation ne serait accomplie selon la précédente procédure, l'émir désignera au moins trois des descendants de feu Mubarak al-Sabah, et l'Assemblée nationale devra prêter allégeance à l'un d'entre eux en tant que dauphin. Le dauphin devra avoir atteint sa majorité, être sain d'esprit, et être le fils légitime de parents musulmans. ${ }^{3}$

20 Bien qu'aucun parlement koweïtien n'ait jamais remis en cause le choix de l'émir, cette procédure rend les crises de succession beaucoup moins probables, et accorde un certain degré de légitimité populaire au souverain. Compte tenu des luttes internes et des rivalités passées au sein de la famille al-Sabah, ces mécanismes constitutionnels paraissent être conçus pour unifier la famille : pour une part en définissant son rôle, pour l'autre en rendant claire la prédominance de l'émir (notamment sur les questions de succession).

21 La Constitution koweïtienne a donc été conçue en partie pour donner des bases solides au pouvoir de la querelleuse famille royale, afin de renforcer la place de l'émir au sein de la famille et de faciliter une transition sans heurts. Cela peut s'illustrer non seulement au travers des dispositions mentionnées plus haut, mais aussi par l'étude des cas où l'émir a écarté des parties fondamentales de ce document. À trois reprises (en 1976, 1986, 1999), l'émir a dissous le Parlement. Les deux premières fois, il a même été plus loin et a suspendu l'Assemblée législative, attribuant ses pouvoirs constitutionnels à son cabinet. La troisième, et plus récente, dissolution fut accomplie de façon constitutionnelle et aboutit à de nouvelles élections parlementaires. En ces trois occasions, les initiatives furent vaguement justifiées par des références aux abus de la démocratie parlementaire. Même si ce n'était pas spécifié, ces abus tournaient autour 
de menaces sur la famille régnante. Non seulement le parlement avait engagé une procédure de retrait de sa confiance à quelques ministres d'al-Sabah, mais il avait aussi, de ce fait, déclenché des rivalités intra-familiales. Les structures établies par la Constitution avaient, dans ces cas, subverti les fins multiples qu'elles avaient pour dessein de servir, et le Parlement (et, lors des deux premières occasions, la Constitution elle-même) paya le prix de la transgression.

\section{Bahreïn}

Pendant des dizaines d'années, Bahreïn fut dans le Golfe l'exact opposé du Koweït en matière de politique. Le Koweït indépendant maintint un niveau élevé de libertés politiques durant de longues périodes de son histoire; Bahreïn était souvent cité comme le système politique le moins libéral du Golfe. Au Koweït, le Parlement fut par deux fois suspendu, mais revint à chaque fois ; à Bahreïn, la suspension du Parlement devint la règle. Le Koweït $a$, dans une certaine mesure, évolué pour intégrer ses citoyens chiites qui restent durement exclus à Bahreïn. Ces différences se sont estompées depuis que le cheikh Hamad est parvenu au trône en 1999 (et a choisi de porter le titre de roi plutôt que celui d'émir). Bahreïn a fait des pas hésitants, mais néanmoins remarquables, vers la libéralisation politique, et fait revivre des pratiques constitutionnelles oubliées.

Cependant, même durant la période pendant laquelle la politique bahreïni était animée d'un esprit très nettement non constitutionnel, les dispositions sur la succession restèrent en vigueur. L'émir bahreïni promulgua une Constitution lors de l'indépendance en 1971 ; comme la Constitution koweïtienne, elle mettait en place un parlement élu. La patience de l'émir avec les parlements s'est avérée beaucoup plus courte que celle de son homologue koweïtien : il a dissous le Parlement en 1975 et nommé une nouvelle assemblée consultative.

Pourtant, la Constitution bahreïni s'est avérée viable dans un domaine particulier : la succession. La question de la succession est résolue clairement à l'accession du nouvel émir, par la désignation de son héritier mâle le plus âgé. La Constitution koweïtienne a recours à la ratification parlementaire pour placer la question de la succession en dehors de tout conflit; Bahreïn use d'un dispositif différent pour le même effet. L'article 1 de la Constitution stipule :

Le trône de Bahreïn est héréditaire. Il se transmet de son Altesse le cheikh 'Isâ bin Salmân al-Khalîfa à son fils aîné, puis au fils aîné de celui-ci, et ainsi de suite, de génération en génération, à moins que, de son vivant, l'émir nomme l'un de ses fils autre que l'aîné comme successeur, et ce conformément au Décret sur la succession prévu à l'alinéa suivant. ${ }^{4}$

L'inscription d'un modèle spécifique dans le texte constitutionnel exclut la succession de toute rivalité familiale. De plus, les deux mécanismes favorisent l'émir : au Koweït, en lui permettant de choisir le candidat (en général, après consultation des membres de la famille, et avec la politique familiale très présente à l'esprit), à Bahreïn, en suivant la lignée.

Tous les doutes sur l'efficacité de la solution bahreïni disparurent à la mort, en 1999, du seul émir à avoir gouverné Bahreïn depuis l'indépendance. Son fils aîné lui succéda immédiatement, en dépit du fait qu'un autre membre de la famille royale avait officié 
en tant que Premier ministre. Bien que ce Premier ministre fût plus âgé et beaucoup plus expérimenté que le prince héritier, la succession ne fut pas contestée.

Le Koweït et Bahreïn se sont écartés quelque peu des normes régionales dans leur utilisation des dispositifs constitutionnels. Le Koweït l'a fait en impliquant les représentants du peuple dans le choix du successeur; Bahreïn, en ayant recours à la primogéniture. Les exemples koweïtien et bahreïni peuvent en partie expliquer la fièvre constitutionnelle qui a saisi les monarchies du Golfe dans les années 1990. En 1992, l'Arabie saoudite honora une promesse faite il y a environ soixante ans d'adopter une loi fondamentale. En 1996, Oman devint le dernier État du Golfe à publier un tel document. La même année, les Émirats arabes unis firent de la Constitution temporaire publiée en 1971 un document permanent. Le Qatar amenda son «Statut temporaire révisé de gouvernement " pour transférer une partie des pouvoirs du Premier ministre à l'émir. En 1998, l'émir du Qatar promit une nouvelle constitution au pays, et nomma un comité en juillet 1999 pour rédiger un texte.

Les initiatives constitutionnelles furent souvent saluées comme des réformes de libéralisation douce. Cette évaluation n'était pas seulement répandue en Occident mais aussi dans la région. Ahmad al-Sa'dun, alors président du Parlement koweïtien (dont les membres élus sont bien conscients de l'absence d'homologues dans la région) proclama que le nom de l'émir du Qatar serait écrit «en lettres d'or ", à la suite de sa promesse d'une nouvelle constitution ${ }^{5}$. Étant donné que la rédaction du document a pris trois ans, et qu'il n'a pas encore été publié (encore moins promulguéb), ce jugement enthousiaste semble prématuré. Mais d'autres pays ont publié des documents. La lecture des textes promulgués tempère souvent l'enthousiasme, car les constitutions contiennent généralement peu de caractéristiques libérales, et les éléments libéraux existants peuvent être facilement contournés. Pourtant, on ne peut considérer ces changements constitutionnels comme de simples engagements rhétoriques. Ces nouvelles constitutions cherchent clairement à éviter les crises de succession, souvent en renforçant le pouvoir du monarque existant. On peut l'observer très clairement dans le cas du Qatar et de l'Arabie saoudite.

\section{Qatar}

Les émirs du Qatar ont été renversés deux fois par leurs successeurs désignés. La seconde fois, en 1995, l'usurpateur était le propre fils de l'émir. Depuis 28 ans, le pays n'a pas connu de succession régulière.

La Constitution actuelle du Qatar - en vigueur jusqu'à la publication et la promulgation de la nouvelle - est appelée "Statut temporaire révisé de gouvernement». Ses dispositions relatives à la succession furent utilisées pour désigner l'émir actuel comme héritier du trône, avant qu'il n'amende le texte. L'ancienne, comme la nouvelle version, permettent à l'émir de désigner un successeur au sein de la famille royale. Elles ne posent toutefois aucune limite réelle à son choix. La désignation du prince héritier est (comme au Koweï) accompagnée d'une part importante de délégation: les princes héritiers ont servi comme premiers ministres et ont souvent été plus impliqués dans les décisions au jour le jour que l'émir. Cependant c'est l'émir qui désigne son successeur. L'article 21 de la Constitution du Qatar prévoyait à l'origine :

Le pouvoir dans l'État est héréditaire au sein de la famille Âl Thanî. Le dauphin sera nommé un an au plus après l'adoption de ce statut temporaire de gouvernement, et sa nomination sera faite par décret émiral après consultation des notables [ceux qui 
lient et délient, ahl al-hall wa-l-caqd, généralement compris comme se composant des clercs et responsables religieux musulmans] dans le pays, et l'accord de la majorité d'entre eux sur cette nomination.

En 1995, la deuxième phrase fut supprimée et la disposition suivante insérée à la place :

Il [le pouvoir dans l'État] est transmis du père à l'un des ses fils, et, à défaut de fils, à celui que l'émir choisit dans la famille Âl Thanî. L'émir désigne le prince héritier par une ordonnance princière après consultation des notables dans le pays [ceux qui lient et délient dans le pays] et avec l'approbation de la majorité d'entre eux. ${ }^{7}$

32 En résumé, le prince héritier exerce souvent une autorité considérable, mais doit sa position au choix de son prédécesseur. Le résultat a souvent été d'entretenir jalousies et rivalités entre l'émir (qui se considérait comme le souverain légitime) et le prince héritier (qui se considérait comme le souverain effectif). En 1972 et 1995, le prince héritier résolvait cette rivalité en déposant l'émir pendant qu'il se trouvait en villégiature. Dans le premier cas (et très probablement dans le second), l'initiative était préventive : le prince héritier déposa l'émir au moment où l'on soupçonnait ce dernier d'envisager de désigner un nouveau prince héritier. L'absence de mécanismes de succession clairs et solides n'a pas conduit aux violences physiques auxquelles faisait allusion Montesquieu (même si un contre coup d'État manqué en 1996 fit courir le risque de violences plus étendues), mais il a abouti à de graves divisions familiales.

L'actuel émir du Qatar a montré qu'il avait retenu la leçon que son père aurait dû connaître. Premièrement, l'émir concentre un plus grand pouvoir, ce qui restreint la possibilité que le prince héritier/Premier ministre n'émerge aussi puissamment que dans le passé. En fait, au moins jusqu'à présent, les postes de prince héritier et de Premier ministre ont été séparés. Deuxièmement, l'étendue de la rivalité a été limitée par la priorité donnée aux fils de l'émir pour la succession. Troisièmement, l'émir a promis une nouvelle constitution. La lenteur du travail du comité, la méthode retenue pour sélectionner ses membres (nomination royale directe), et sa réticence à publier ses projets - avant révision (et peut être modification) - montre que le processus a été très étroitement surveillé et contrôlé. Tout indique, alors, que le but est moins la libéralisation que la normalisation du pouvoir. Le changement peut néanmoins encore être réel; dans les termes oubliés du discours politique classique et libéral, le Qatar semble évoluer lentement du despotisme vers une monarchie.

\section{Arabie saoudite}

$34 \mathrm{Au}$ moment de l'unification du royaume, les souverains d'Arabie saoudite promirent qu'une loi fondamentale serait publiée afin d'organiser le système politique. Quatre rois se succédèrent sur le trône, avant qu'un document soit publié. Longtemps considérée comme une réforme de libéralisation politique, la loi fondamentale, lorsqu'elle fut finalement publiée, surprit la plupart de ses lecteurs, qui jugèrent qu'elle n'était pas à la hauteur de leurs attentes. Même selon les standards des documents constitutionnels arabes, les dispositions en faveur des droits et libertés étaient très maigres. Les impôts ne pouvaient être imposés que par la loi, mais puisque les lois étaient simplement promulguées par décret, cela n'imposait aucune limite. L'État était chargé d'offrir l'éducation, les soins de santé et l'emploi, ce qu'il faisait déjà et ferait bientôt bien moins (à cause de la crise fiscale). Le document prévoyait aussi la mise en place d'un conseil consultatif ainsi qu'un budget, mais fournissait peu d'indications sur la manière dont ces mesures devaient être mises en œuvre (un conseil consultatif fut 
effectivement nommé). La Constitution contenait de nombreuses références, non seulement à l'islam, mais à la sharîa islamique, et si des limites à l'autorité politique figuraient dans le document, c'est uniquement là qu'on pouvait les trouver. politiques n'ont jamais produit d'anciens présidents. Les présidents officient à vie ou jusqu'à ce qu'ils soient évincés de leur charge (un événement de plus en rare). Dans certains pays (en Irak et manifestement en Syrie), aucune succession constitutionnelle régulière ne s'est produite sur plus d'une génération; dans d'autres républiques (la Tunisie, par exemple), cela n'a jamais eu lieu. 

lairement un successeur, le président tunisien Bourguiba, opéra un choix trop efficace: Zine al-Abidine Ben Ali utilisa de vagues dispositions constitutionnelles sur l'incapacité du président pour déloger son patron de sa fonction. elles soulèvent d'autres questions : pourquoi les présidents arabes ne suivent-ils pas la même logique que les monarchies arabes ? Pourquoi les présidents arabes semblent-ils désireux d'emporter leurs opinions sur la succession dans la tombe, alors que les monarques arabes font de la désignation d'un successeur un de leurs premiers actes? Quels types de calculs sont faits par les deux catégories de gouvernants? 

situation beaucoup plus restreinte en ce qui concerne leurs successeurs. Rois et émirs ne peuvent se tourner que vers un groupe étroit de membres de la famille royale. Même si les membres dirigeants peuvent avoir des inclinaisons politiques connues ou disposer de bases institutionnelles, le choix d'un individu particulier n'implique généralement pas le même degré d'engagement politique. La tradition guide encore davantage le choix : en Arabie saoudite, la succession a été, à ce jour, restreinte à un groupe étroit parmi les fils de 'Abd al-cAziz. Les émirs du Koweït ont généralement (mais pas toujours) alterné entre deux branches de la famille royale. La primogéniture n'est pas formellement établie au Qatar, mais les fils de l'émir actuel sont automatiquement de très bons candidats.

Deuxièmement, les monarques du Golfe sont bien davantage en mesure de garantir la place de leur successeur. Accepter le poste de prince héritier implique de reconnaître que la charge du souverain régnant est une charge à vie. Dans la mesure où les constitutions républicaines ne reconnaissent pas les charges à vie, le successeur désigné d'un président ne prononce pas un tel engagement. De plus, les dispositifs constitutionnels décrits plus haut, tels que la primogéniture ou la ratification parlementaire d'un candidat, peuvent assurer les successeurs des souverains. Il s'ensuit que ces successeurs sont bien mieux protégés et, par conséquent, bien moins susceptibles d'agir pour hâter la succession.

\section{Conclusion : les usages des constitutions}

En 1952, le groupe de responsables politiques entourant la monarchie jordanienne se trouva face à un énorme problème. L'ordre politique qu'ils soutenaient était centré autour d'un roi, mais celui qui occupait alors le trône, Talal, présentait des signes graves d'incapacité mentale. La situation n'était pas totalement imprévue. En 1951, une nouvelle constitution avait été rédigée à l'ombre de la maladie de Talal; ses auteurs y avaient inclus des clauses de succession claires. L'article 28 autorisait la déposition du roi :

Dans le cas où le roi serait incapable par suite d'une maladie mentale, le Conseil des ministres, sur confirmation de sa maladie, convoquera immédiatement l'Assemblée nationale. Si la maladie est confirmée de façon définitive, l'Assemblée nationale déposera le roi par résolution, par quoi le titre au trône passera après lui à l'ayant droit, selon les dispositions de la Constitution. Si la Chambre des députés est dissoute à ce moment ou si son mandat a pris fin et qu'aucune nouvelle Chambre n'a été élue, l'ancienne Chambre sera convoquée dans ce but. ${ }^{11}$

Utiliser ces dispositions ne fut pas chose aisée; cela exigeait que les responsables jordaniens persuadent le Parlement de la nécessité d'opérer un changement. Ils se tournèrent vers un ancien ministre de la Santé, l'un des meilleurs médecins du pays, pour qu'il effectue l'expertise qui amena finalement le Parlement à porter Hussein, le fils de Talal, sur le trône.

Dans les années 1980, les rumeurs concernant les capacités décroissantes du président tunisien Bourguiba s'amplifièrent. La Constitution tunisienne donnait peu d'indications sur la manière de faire face à une telle situation. Dans une certaine mesure, des amendements récents rendaient la situation encore plus délicate, en supprimant toute limitation de durée du mandat. Certes, il y avait des clauses de succession, la question 
avait déjà suscité une certaine controverse dans le passé12. L'assemblée constituante élue et les parlements avaient généralement privilégié la désignation du président du Parlement, alors que les gouvernements tunisiens souhaitaient désigner l'un de leurs membres. Les partisans du Parlement, qui perdirent la plupart des batailles dans les développements constitutionnels tunisiens, perdirent aussi ce combat, au motif que le système tunisien était présidentiel, et que tout rôle du parlement empiétait donc sur l'exécutif. On ne mesura les implications pratiques de ces débats que lorsqu'en 1987, Zine el-Abidine Ben Ali, le Premier ministre de Bourguiba, proclama que la sénilité du président l'empêchait de continuer à assumer sa fonction. Ben Ali invoqua une vague disposition constitutionnelle mentionnant l'incapacité du président; cette disposition ne faisait pas mention de la personne autorisée à émettre un tel jugement. Alors que les responsables jordaniens agirent conformément aux dispositions constitutionnelles, certes sommaires, mais précises, les actions de Ben Ali équivalaient à un coup d'État. En réalité, il ne se tourna pas vers le Parlement, mais vers son propre pouvoir pour décider; il n'obtint qu'un bulletin médical imprécis pour étayer l'affirmation selon laquelle Bourguiba était incapable d'accomplir les devoirs de la présidence ${ }^{13}$.

51 Le mécanisme jordanien était beaucoup plus difficile à utiliser que le tunisien, mais il offrait davantage de continuité et de légitimité. Les constitutions ont parfois leurs coûts, mais elles ont aussi leurs bénéfices: elles offrent stabilité, clarté et des procédures précises. Les monarchies arabes - spécialement celles des États du Golfe ont fait grand usage des vertus des constitutions dans les processus de succession.

L'argument présenté dans cet article est très modeste. Il ne prétend pas que les monarchies arabes s'approchent de l'idéal libéral de l'État de droit (dans de nombreux domaines, les républiques arabes en sont beaucoup plus proches que les monarchies). Il n'a pas non plus une vision apologétique des monarchies arabes. Au contraire, il tente de ne pas limiter l'étude des institutions politiques arabes à une concentration excessive sur les idéaux de la démocratie libérale. Les monarchies arabes - comme les républiques arabes - ne sont pas des démocraties libérales. Une telle constatation permet peut-être d'amorcer un début de compréhension de leur nature (et peut-être même pas). Parce que les monarchies constitutionnelles ne sont ni libérales ni démocratiques, elles sont souvent rejetées par les spécialistes comme non pertinentes pour la science politique.

53 Si nous cherchons à comprendre ce qu'est la politique arabe - et pas seulement ce qu'elle n'est pas - alors de tels textes deviennent plus significatifs. Les constitutions arabes ont leurs finalités, et l'une des principales - dans les monarchies - est de renforcer l'autorité du souverain au sein la famille royale, et de fournir des mécanismes clairs pour des successions non disputées. En un sens, de telles constitutions ne servent pas à limiter l'autorité du chef de l'État mais à la renforcer; les monarques arabes sont devenus de plus en plus ingénieux pour combiner mécanismes juridiques et pouvoir familial. 


\section{BIBLIOGRAPHIE}

Brown Nathan, 1997, The Rule of Law in the Arab World, Cambridge, Cambridge University Press.

-, 2000, «Constituting Palestine : The Effort to Write a Basic Law for the Palestinian Authority », Middle East Journal, n54-1, p. 25-43.

AL-HAKîm Tawfîq, 1998, Al-sultân al-hâ’ir, Le Caire, Maktabat Misr.

HERB Michael, 1999, All in the Family: Absolutism, Revolution, and Democracy in the Middle Eastern

Monarchies, Albany, SUNY Press.

AL-MAZFAR Zuhayr, 1992, 7 Nuvimbir : al-thawra al-hadi'a, (7 novembre : La Révolution tranquille), Tunis, Mu'assasat cabd al-karîm bin cabd allah li-l-nashr wa-l-tawzî̀ .

Montesquieu, 1951, L’Esprit des Lois, Paris, Gallimard, « Pléiade », livre 5, ch. 14.

Przeworski Adam, 1988 , «Democracy as a Contingent Outcome of Conflicts », dans Jon Elster et Rune Slagstad (dirs), Constitutionalism and Democracy, Cambridge, Cambridge University Press.

\section{NOTES}

1. Sur ces systèmes en général, voir Michael Herb (1999). Les opinions de Herb sur les règles de succession diffèrent des miennes : il considère que l'ambiguïté des mécanismes de succession renforce la plasticité des régimes. Je ne partage pas ce point de vue, mais suis d'accord avec son observation que les constitutions ont servi à régler les successions. Je me fonderai sur l'observation que les constitutions de la péninsule arabique procèdent souvent ainsi d'une manière qui clarifie les procédures, et renforce la place du monarque, sans supprimer le rôle de la famille royale (Herb considère que la famille royale joue un rôle critique pour la longévité du régime).

2. J'ai écrit ailleurs sur les textes de la Constitution koweïtienne. Voir The Rule of Law in the Arab World, 1997. L'étude est ici basée sur mon précédent travail et sur des recherches complémentaires, consistant surtout en des entretiens avec les membres survivants de l'Assemblée.

3. Une traduction de la Constitution koweïtienne est disponible sur http: //www.oefre.unibe.ch [consulté en juin 2005].

4. On peut consulter la Constitution de 1973 sur le site http://www.oefre.unibe.ch/law.icl/ ba01000_html. En 2002, cette clause fut amendée pour insérer le nom de Hamad comme successeur de cisâ.

5. Deutsche Press-Agentur, 17 novembre 1998.

6. NDT : la nouvelle Constitution du Qatar est entrée en vigueur en juin 2005.

7. Le texte ancien et sa version amendée peuvent être consultées sur : http ://208.21.175.109/ RelatedArticlesGvnSPName.asp?SPName=CHRN\&StructuredIndexCod e=0\&LawBookID=021020011648619\&Year1=\&Year2=\&YearGorH=, [consulté le 10 janvier 2003].

8. On peut consulter ce texte à l'adresse : htttp://www.saudinf.com/main/c541.html. [consulté en juin 2005].

9. La Constitution prévoit des successeurs intérimaires - le président du Parlement et le président de la Haute Cour constitutionnelle. Mais le président par intérim ne peut pas se présenter aux élections présidentielles. En 1981, Hosni Moubarak ne succéda pas à Anouar alSadate avant que le parlement ne se soit prononcé. 
10. J'ai traité de la loi fondamentale palestinienne dans «Constituting Palestine: The Effort to Write a Basic Law for the Palestinian Authority ", 2000.

11. On peut consulter le texte: http://www.kinghussein.gov.jo/constitution_jo.html. [consulté en juin 2005].

12. L'histoire de ce débat est couverte de façon très extensive dans un essai de Zuhayr al-Mazfar, président du Conseil constitutionnel, imprimé sous : 7 Nuvimbir : al-thawra al-hadi'a, 1992.

13. La déclaration de Ben Ali est reproduite dans $i b i d$.

INDEX

Mots-clés : Constitution, monarchie constitutionnelle, succession politique

\section{AUTEURS}

NATHAN BROWN

Université George Washington 